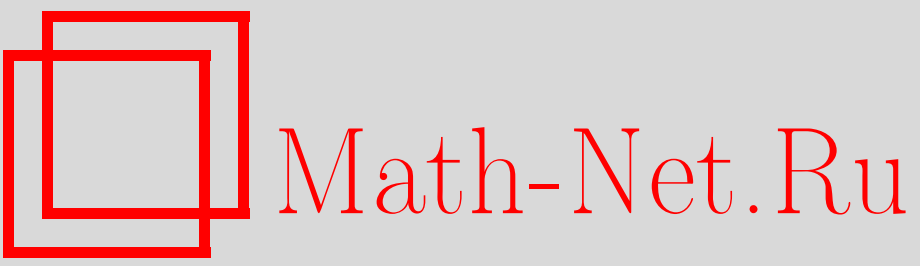

М. Бакурадзе, М. Джибладзе, В. В. Вершинин, Характеристические классы и трансферные соотношения в кобордизмах, УМH, 2001, том 56, выпуск 3, 157-158

DOI: https://doi.org/10.4213/rm400

Использование Общероссийского математического портала Math-Net.Ru подразумевает, что вы прочитали и согласны с пользовательским соглашением

http://www.mathnet.ru/rus/agreement

Параметры загрузки:

IP: 35.173 .137 .237

26 апреля 2023 г., 10:24:53 


\title{
ХАРАКТЕРИСТИЧЕСКИЕ КЛАССЫ И ТРАНСФЕРНЫЕ СООТНОШЕНИЯ В КОБОРДИЗМАХ
}

\author{
М. БАКУРАДЗЕ, М. ДЖИБЛАДЗЕ, В. В. ВЕРШИНИН
}

Большинство соотношений для элементов конечного порядка в колшце симплектических кобордизмов между элементами Рея $\varphi_{i}[1]$ и свободными образующими в размерностях до 32 [2] могут быть условно разбиты на три типа: первому типу соответствуют соотношения, которые следуют, главным образом, из соотношений в целочисленной части $M S p_{4 n}$. Более детально, соотношения первого типа имеют вид $(x+y) \varphi_{i}=0$ и следуют из того факта, что сумма свободных образующих $x+y$ делится на 2 , тогда как элементы Рея имеют порядок 2 . Соотношения второго типа имеют вид $z \varphi_{i}=0$, где $z$ снова есть $4 n$-мерная образующая свободной части. Что касается соотношений третьего типа, то оказьвается, что они могут быть включены в серию соотношений следующей природы: большинство $4 n$-мерных образующих с малыми $n$ после умножения на элементы Рея $\varphi_{i}$ для всех $i \geqslant 0$ принадлежат идеалу, порожденному элементами Рея малых размерностей.

Пусть $\zeta$ есть универсальное $S p(1)$-расслоение, тогда $\zeta_{1} \otimes_{\mathbb{C}} \zeta_{2} \otimes_{\mathbb{C}} \zeta_{3}$ есть симплектическое расслоение над $B S p(1) \times B S p(1) \times B S p(1)$. Рассмотрим первый симплектический класс Понтрягина-Коннера-Флойда

$$
p_{1}\left(\zeta_{1} \otimes_{\mathbb{C}} \zeta_{2} \otimes_{\mathbb{C}} \zeta_{3}\right)=\sum a_{k l m} p_{1}^{k}\left(\zeta_{1}\right) p_{1}^{l}\left(\zeta_{2}\right) p_{1}^{m}\left(\zeta_{3}\right) .
$$

Значения гомоморфизма Гуревича $h: \varphi_{*}(M S p) \rightarrow H_{*}(M S p)=Z\left[q_{1}, q_{2}, \ldots,\right]$ на коэффиициентах $a_{k l m}$ изучались в [3]. В малых размерностях имеем $h\left(a_{100}\right)=h\left(a_{010}\right)=h\left(a_{001}\right)=4$;

$h\left(a_{200}\right)=h\left(a_{020}\right)=h\left(a_{002}\right)=0$;

$h\left(a_{110}\right)=h\left(a_{101}\right)=h\left(a_{011}\right)=24 q_{1} ;$

$h\left(a_{111}\right)=360 q_{2}$;

$h\left(a_{210}\right)=\cdots=h\left(a_{012}\right)=60 q_{2}-24 q_{1}^{2}$

$h\left(a_{300}\right)=\cdots=h\left(a_{003}\right)=0 ;$

$h\left(a_{220}\right)=\cdots=h\left(a_{022}\right)=280 q_{3}-120 q_{1} q_{2}+24 q_{1}^{3} ;$

$h\left(a_{310}\right)=\cdots=h\left(a_{013}\right)=112 q_{3}-96 q_{1} q_{2}+48 q_{1}^{3}$;

$h\left(a_{211}\right)=\cdots=h\left(a_{112}\right)=1680 q_{3}-360 q_{1} q_{2}$;

$h\left(a_{122}\right)=\cdots=h\left(a_{221}\right)=75600 q_{4}-3360 q_{1} q_{3}+360 q_{1}^{2} q_{2} ;$

$h\left(a_{410}\right)=\cdots=h\left(a_{014}\right)=180 q_{4}-360 q_{1} q_{3}+420 q_{1}^{2} q_{2}-120 q_{2}^{2}-120 q_{1}^{4}$.

Аналогичные вычисления значений гомоморфизма Гуревича на образующих $z_{i}$ малшх размерностей $M S p_{4 n}$ дают выражение $z_{i}$ в терминах коэффициентов $a_{k l m}$. Нашим основным результатом является следующая теорема.

Теорема. В терминах коэффициентов $a_{k l m}$ структура $M S p_{4 k}, k \leqslant 4$, может быть проинтерпретирована следующей таблищей:

\begin{tabular}{|c|c|c|}
\hline$k$ & $M S p_{4 k}$ & образующие \\
\hline 1 & $Z$ & $a_{011}$ \\
\hline 2 & $Z+Z$ & $a_{012}, a_{111}$ \\
\hline 3 & $Z+Z+Z$ & $a_{022}, a_{011} a_{111}, a_{211}$ \\
\hline 4 & $\oplus^{5} Z$ & $a_{014}, a_{011} a_{211} \cong a_{012} a_{111}, a_{122}, a_{111}^{2}, 2 y_{4}$ \\
\hline
\end{tabular}

Для всех $i \geqslant 0$ имеем

a) $\varphi_{i} a_{011}=\varphi_{i} a_{012}=\varphi_{i} a_{022}=\varphi_{i} a_{014}=0$,

b) $\varphi_{i} a_{111}$ и $\varphi_{i} a_{122}$ принадлежат идеалу $\varphi_{0} M S p^{*}$;

c) $\varphi_{i} a_{211}$ принадлежат идеалу $\varphi_{0} M S p^{*}+\varphi_{1} M S p^{*}$.

Работа первого автора поддержана CRDF (грант GM1-2083);

Работа второго автора поддержана INTAS (грант 93-2618-EXT) и TMR (грант ERB FMRX CT-97-0107);

Работа третьего автора поддержана РФФИ (грант № 99-01-00574). 
Доказательство теоремы использует резултаты работ [1]-[11] и, главным образом, основывается на предложениях, приведенных ниже. Пусть $\xi$ есть универсальное $U(1)$-расслоение, $\Lambda$ есть универсалшное $\operatorname{Spin}(3)$-расслоение. Тогда расслоение $\pi: B U(1) \rightarrow B S p(1)$ является сферическим пучком, соответствующим $\Lambda$, и $\pi^{*}(\zeta)=\xi+\bar{\xi} ; \pi^{*}(\Lambda)=\xi^{2}+R ; \zeta \otimes_{H} \zeta=\Lambda+R$. Пусть $N$ есть нормализатор тора $U(1)$ в $S p(1)$. Расслоение $p: B N \rightarrow B S p(1)$ совпадает с проективизацией расслоения $\Lambda$, и мы имеем каноническое расщепление $p^{*}(\Lambda)=\mu+\nu$, определенное проективизацией $p$, где $\mu$ и $\nu$ суть двумерное и линейное вещественные расслоения. Для двулистного накрытия $q: B U(1) \rightarrow B N$ имеем $q^{*}(\mu)=\xi^{2}$ и $q^{*}(\nu)=R$. Пусть $\tau(\pi)$ и $\tau(p)$ суть отображения трансфера [4] расслоений $\pi$ и $p$.

ПреДЛОЖЕНИЕ 1. Имеем: $\pi^{*} \tau(\pi)^{*}=1+I^{*} u \pi^{*} \tau(p)^{*}=q^{*}$.

Это предложение следует из резултатов работы [5].

Из определения трансфера Атьи вытекает следующий факт.

ПреДлОЖениЕ 2. Для трансфера Атьи! двулистного накрытия $1 \times q: B U(1) \times$ $B U(1) \rightarrow B U(1) \times B N$ имеем

$$
\left(\xi_{1} \xi_{2}^{2}+\bar{\xi}_{1} \bar{\xi}_{2}^{2}\right) !=\left(\xi_{1}+\bar{\xi}_{1}\right) \otimes_{\mathbb{R}} \mu .
$$

Пусть $f$ есть отображение $f: B N \rightarrow B Z_{2}$, индуцированное проекцией $N$ на группу Вейля $Z_{2}$, и пусть $\tau(1 \times q)^{*}$ есть гомоморфизм трансфера для приведенного выше двулистного накрытия $1 \times q$.

ПРЕДЛОЖЕНИЕ 3. Существуют әлементы $\gamma_{i}$ в $M S p^{*}(R P(\infty))$, для которых выполняется следующая формула

$$
\tau(1 \times q)^{*}\left(p_{1}\left(\xi_{1} \xi_{2}^{2}+\bar{\xi}_{1} \bar{\xi}_{2}^{2}\right)\right)=p_{1}\left(\left(\xi_{1}+\bar{\xi}_{1}\right) \otimes_{\mathbb{R}} \mu\right)+\sum_{i \geqslant 0} f^{*}\left(\gamma_{i}\right) p_{2}^{i}\left(\left(\xi_{1}+\bar{\xi}_{1}\right) \otimes_{\mathbb{R}} \mu\right) .
$$

Доказательство следует из результатов работы [6] и предыдущего предложения.

Из [7] и [8] получаем следующий факт.

ПреДЛОЖЕНИЕ 4. Для всех $a \in M S p^{*}(B U(1))$ имеем $\varphi_{j} \tau(\pi)^{*}(a)=0$.

Соединяя результаты предыдущих предложений, получаем следующее утверждение.

ПреДЛОЖЕНИЕ 5. В $M S p^{*}(H P(4) \times H P(4))$ следующие соотношения справедливь для всех $j \geqslant 0$ :

a) $\varphi_{0} p_{1}\left(\zeta_{1}\right)+\varphi_{1} p_{1}^{2}\left(\zeta_{1}\right)+\varphi_{2} p_{1}^{4}\left(\zeta_{1}\right)$ делum $\varphi_{j} p_{1}\left(\zeta_{1} \otimes_{\mathbb{C}} \zeta_{2}^{2}\right)$

b) $\varphi_{j} p_{1}\left(\zeta_{1} \otimes_{\mathbb{R}} \zeta_{2}\right)=0$.

ЗАмечАнИЕ. В размерности 32 имеется элемент $y_{4}^{2}$ такой, что $\varphi_{2} y_{4}^{2}$ не принадлежит идеалу, порожденному $\varphi_{0}$ и $\varphi_{1}$. Более того, $\varphi_{2}{ }^{i} y_{4}^{2}$ не принадлежит идеалу, порожденному $\varphi_{0}, \varphi_{1}, \ldots$, $\varphi_{2^{i-1}}, i \geqslant 1$

\section{СПИСОК ЛИТЕРАТУРЫ}

[1] N. Ray // Topology. 1971. V. 10. P. 261-270. [2] В. В. Вершинин // Сиб. матем. журн. 1983. T. 24. C. 50-63. [3] M. Imaoka // Hiroshima Math. J. 1982. V. 12. № 1. P. 151-181. [4] J. C. Becker, D.H. Gottlieb // Topology. 1975. V. 14. P. 1-12. [5] M. Feshbach // Trans. Amer. Math. Soc. 1979. V. 251. P. 139-169. [6] F. W. Roush. Transfer in Generalized Cohomology Theories. Budapest: Akadimiai Kiady, 1999. [7] В. Г. Горбунов // Матем. сб. 1990. T. 181. C. 506-520. [8] J. M. Boardman. Stable Homotopy Theory. University of Warwick, 1966. [9] М. Бакурадзе, Р. Надирадзе // Труды Тбилис. матем. ин-та. 1991. Т. 94. С. 12-28. [10] В. М. Бухштабер // Итоги науки и техники. Совр. пробл. матем. Т. 10. М.: ВИНИТИ, 1977. C. 5-178. [11] A. Dold // Math. Z. 1976. V. 148. P. 215-244. 